MATEC Web of Conferences 22,02006 (2015)

DOI: $10.1051 /$ matec conf/ 20152202006

(C) Owned by the authors, published by EDP Sciences, 2015

\title{
Deployment Strategy for Charging Piles Based on Distribution Network Capacity Planning and Users' Needs
}

\author{
Chongyang Du \& Bing Qi \\ North China Electric Power University, Beijing, China \\ Hongwei Xie \\ East Inner Mongolia Electric Power Company Limited, Huhhot, Inner Mongolia, China \\ Hongchang Lv \\ Beijing Sifang Automation Co., Ltd., Beijing, China
}

\begin{abstract}
Electric vehicles are the most potential transports in the future. However, the large scale of charging facilities will make a great influence on gird. There is a need to make a research on the construction of charging facilities. Based on the power demand characteristics of electric vehicle charging, distribution network capacity, charging system performance and other aspects, this paper mainly researched the deployment strategy of charging piles. First, the authors built up a model with characteristics of charging power demand of electric vehicle and a model of charging service system. The characteristic of daily load curve is analyzed. Second, based on these works, the authors designed the progress of strategy making. At last, the progress was verified by the actual use case.
\end{abstract}

Keywords: distribution capacity; service performance; deployment strategy; load characteristics

\section{INTRODUCTION}

As a new generation of means of transportation, electrical vehicles have incomparable advantages in energy conservation, and they can reduce human's dependence on traditional fossil fuels. With the increase in the number of electric vehicles, the scale of the charging facilities will follow the rapid increase. When the charging piles have access to grid in a large scale, it will exert great impacts on the safe operation of the power grid. So no matter how many domestic or abroad researchers there are, both of them have made a lot of research on facilities construction and management, including the charging station location, the charging guide strategies and so on. In paper [1], it works out a planning model for the charging station by analyzing and forecasting the charging demand of electric vehicles. In paper [2], based on the capacity configuration of substation, it was used to study the charging access control mode of electric vehicles, and aim at reducing the impact of electric vehicles that have access to the grid during the peak period by optimizing the scheduling policy. However, these researches are only considered from the perspective of grid planning. They do not take full account of the user experience of electric vehicles. Though they are effective in controlling the impact of electric cars on the grid, they may make the system hard to meet the needs of users. In this paper, based on the load characteristics of electric vehicles, we researched the deployment strategy of charge piles which is able to meet the basic needs of users in an area with given power distribution capacity. Through these researches, we can provide a reference for the deployment of charging piles and the distribution of grid capacity planning in the future.

\section{MODEL BUILDING FOR THE DEPLOYMENT STRATEGY}

The deployment strategy for charging piles is aimed to obtain the optimum number of deployment which is known as the base load curves of local electricity consumption, the power demand of electric vehicles and the power distribution capacity of substation. The following assumptions are worked for the aim ${ }^{[3]}$ :

(1) Electronic vehicles are charged when they finish the last driving of the day;

(2) The Charging of electric vehicles is in VOG mode, which means that there is no access control for charging ${ }^{[4]}$;

(3) The charging power of the pile is considered as a fixed value.

In order to develop the strategy for the deployment of charging pile, the first step is to acquire the basic data used for the analysis, including load curves of electric cars, daily load curves of local electricity consumptions and the distribution capacity of local substations. To acquire these data, it needs to build up the models. The process of the model building is shown as follows. 
2.1 Model of the electric vehicle's power demand characteristics

The charging load of electric vehicle is determined by various factors, mainly including the initial SOC, the duration for charging and the starting point of charging ${ }^{[5]}$.

The initial SOC is determined by daily mileage of the electric car. According to the survey results in paper [1] in the mileage of a one-day trip, one-day trips within $100 \mathrm{~km}$ mileage are more than $90 \%$, and other situations that more than $50 \%$ are within the $50 \mathrm{~km}$. The probability distribution of mileage is shown in Figure 1:

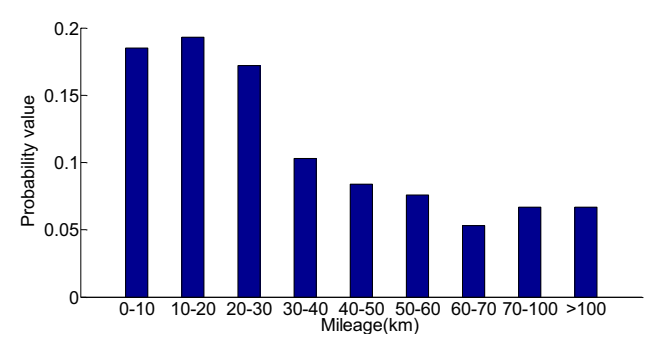

Figure 1. Probability distributions of daily mileage

The curve of the distribution is approximately the curve of log-normal function, which can be fitted by the log-normal distribution function. Thus, we can get a fitting function for daily mileage of electric cars through it ${ }^{[6]}$ :

$$
f_{D}(x)=\frac{1}{x \sigma_{D} \sqrt{2 \pi}} \exp \left[-\frac{\left(\ln x-\mu_{D}\right)^{2}}{2 \sigma_{D}^{2}}\right]
$$

We set E0 as the value of initial SOC, the relationship between E0 and mileage D is shown as follows:

$\mathrm{E} 0=1-\mathrm{d} / \mathrm{R}$

Where $\mathrm{R}$ is the maximum mileage of an electric vehicle, we set it $200 \mathrm{~km}$ in this paper. Thus, we can obtain the probability distribution density function of initial SOC:

$$
f_{E_{0}}=\frac{1}{R\left(1-E_{0}\right) \sigma_{D} \sqrt{2 \pi}} \bullet \exp \left[\frac{-\left(\ln \left[R\left(1-E_{0}\right)\right]-\mu_{D}\right)^{2}}{2 \sigma_{D}^{2}}\right]
$$

$$
\text { Where, } \mu_{D=3.6}, \sigma_{D=0.91} \text {. }
$$

The relationship between the charging duration length $\mathrm{T}$ and the initial SOC is shown as follows:

$$
T=\frac{\left(1-E_{0}\right) S}{p_{0}}
$$

Where $\mathrm{S}$ is the battery capacity of electric vehicles, $\mathrm{p} 0$ is the charging power of electric vehicles.

Electric vehicles will be charged after the whole trip is ended. According to the reference [1], the distribution function of charging start time is shown as fol- lows ${ }^{[7]}$ :

$f_{s}(x)=\left\{\begin{array}{l}\frac{1}{\sigma \sqrt{2 \pi}} \exp \left(-\frac{x-\mu}{2 \sigma^{2}}\right), \mu-12<x<24 \\ \frac{1}{\sigma \sqrt{2 \pi}} \exp \left(-\frac{x+24-\mu}{2 \sigma^{2}}\right), 0<x<\mu-12\end{array}\right.$

In this equation, $\mu=17.6, \sigma=3.4$.

According to the model, we can obtain load graph of electric vehicles in a certain scale in the VOG mode through the Monte Carlo simulation. According to the reference [1], the Load curve of electric vehicles will usually begin to rise sharply at 16:00 of the day, and the time to peak demand of charge is about 19:00. After that, it will slowly decline until it's about 4:00 next day to reach the low ebb of the charging demand.

\subsection{Power distribution capacity of substation and daily load-curve}

Changing process of power system load curve is a non-stationary random process; daily load-curve reflects the time-varying characteristics of load demand in a circadian rhythm. Figure 2 shows the average daily load-curve of one household in a certain region [8]:

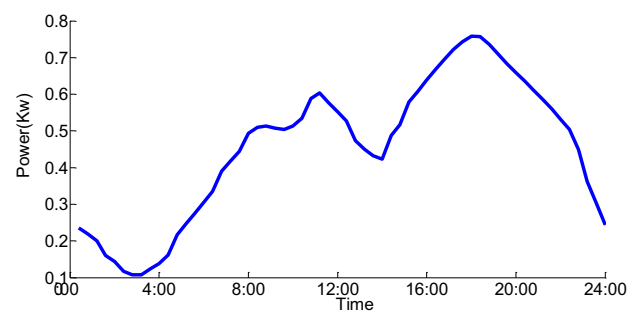

Figure 2. Average daily load curve of each household

As it can be seen from the previous load graphs, the residential electricity load peaked at 20:00. As the analysis shows in 2.1 , the load peak of electric vehicles is about 19:00. When the peak superposition is very serious, the peak superposition will bring tremendous pressure to the distribution network. Therefore, in the case of disorderly charge, the number of the recommended deployment of charging piles must ensure that the peak superposition does not exceed the value of the local distribution's planning capacity ${ }^{[9]}$.

\subsection{Performance of the evaluation model of charging service system}

The model of electric vehicle charging systems based on queuing theory is $\mathrm{M} / \mathrm{M} / \mathrm{C} / \mathrm{N} /{ }^{\infty}$. Now, we assume that the number of charging piles in the system is $\mathrm{s}$, the number of electric vehicles receiving charging service in system is $\mathrm{n}$. According to the basic queuing theory, the operation indexes of $\mathrm{M} / \mathrm{M} / \mathrm{C} / \mathrm{N}$ / model are shown as follows ${ }^{[10]}$.

Load intensity of system $\rho$ : 
$\rho=\frac{\lambda}{\mu}$

Utilization of system $\beta$ :

$\beta=\frac{\lambda}{s \mu}$

The average queue length-L q:

$L_{q}=\frac{\beta(S \beta)^{S} p_{0}}{S !(s-\beta)^{2}}$

$p_{0}=\frac{1}{\sum_{n=0}^{s-1} \frac{\rho^{n}}{n !}+\frac{\rho^{s}}{s !} \frac{1}{s-\rho}}$

Average waiting time-Tq:

$$
T_{q}=\frac{L_{q}}{\lambda}
$$

These indexes mentioned earlier are key indicators to assess the overall operation of queuing system of electric vehicles ${ }^{[11]}$. In this model, $\lambda$ is the access rate to the charging system of electric vehicles in a certain time, and $\mu$ is the number of electric vehicles that the system can service in per time unit. $\mathrm{Tq}$ is the main foundation for the assessment ${ }^{[12]}$.

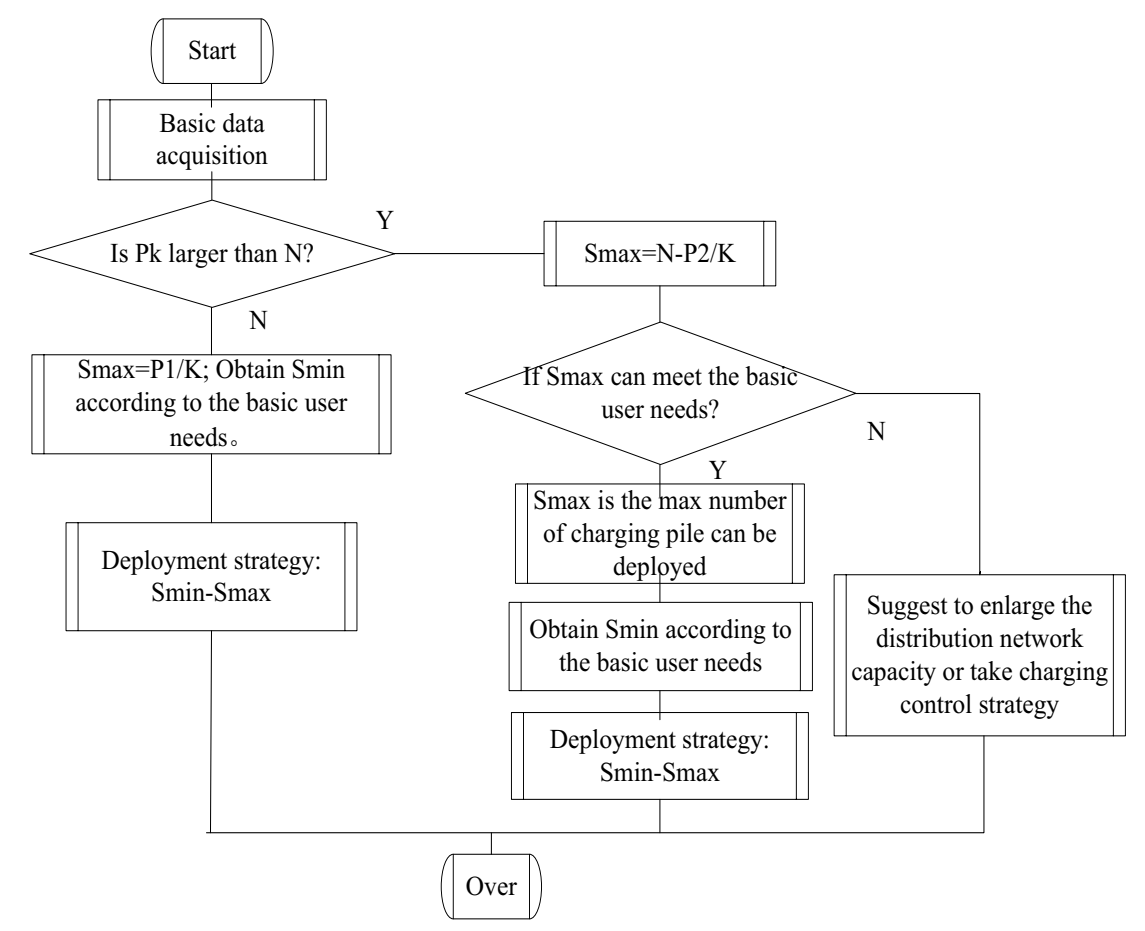
number of charging piles.

\section{PLANNING FOR THE CHARGING PILES TO} BE DEPLOYED

Analysis process described by the SDL diagram is shown in Figure 3.

(1) Preparation for basic data

Based on the analysis model of EV load characteristics in 2.1 and combined with the number of local electric vehicle, we can obtain the load graph of electric vehicles and extract the peak load $\mathrm{P}_{1}$. We can also obtain the access rate $\lambda$ of the electric vehicles in the peak period according to the data of system load curve.

Then, we take the local daily load curves, extract the peak load $\mathrm{P}_{2}$, superimposed the daily load curve of electric vehicles and local power grid load curve, get integrated load curve, and extract the integrated load peak value $\mathrm{Pk}$.

At last, we obtain the local power distribution capacity $\mathrm{N}$ according to the local distribution capacity planning and average charging power $\mathrm{K}$ of the charging piles which are needed to be deployed.

(2) Analysis of the data

We calculate the sum of P1 and P2, and determine whether it is greater than the distribution capacity $\mathrm{N}$.

If it is less than $\mathrm{N}$, then the maximum number of charging pile deployment $S_{\max }$ can be calculated by the peak power demand which is obtained by the electric vehicle charging $\mathrm{P}$ land the average charging power K of piles. According to minimum standards of service, we can obtain the lower limit $S_{\min }$ of the 
If it is more than $\mathrm{N}$, then we can acquire the $\mathrm{S}_{\max }$ : $\mathrm{S}_{\max }=\mathrm{N}-\mathrm{P}_{2} / \mathrm{K}$ (11)

Thus, we can use the evaluation model about the performance of charging service system to assess if the system performance can satisfy the users' needs in case of the $\mathrm{S}_{\max }$. If $\mathrm{S}_{\max }$ can satisfy the needs of users, then we will calculate the $S_{\min }$ based on the formulation (6)-(10) and the index of the basic service. Otherwise, we will suggest increasing the value of the grid planning distribution capacity or adopt the charge control strategy.

According to the analysis results mentioned earlier, we can give the range of scale of the deployment: $\left[\mathrm{S}_{\min }, \mathrm{S}_{\max }\right]$. We can also give suggestions if there is a need to enlarge the capacity of the distribution network.

\section{ANALYSIS OF PRACTICAL USE CASE}

Now, we will analyze the pile deployment strategy for EVs in a substation supply area which is $35 \mathrm{kv}$, and its distribution capacity $\mathrm{N}$ is $7 \mathrm{MW}$. In this area, there are seven residential areas with 12000 residents in total Assume that every resident has 0.75 cars, thus, there are 9000 cars in this area. If 50 percent of these cars are EVs, then we need to make a strategy for 4500 EVs. In this case, we set that the charging power of the piles is $5 \mathrm{KW}$, and the waiting time of the users during peak hour will no more than $20 \mathrm{~min}$. Take $\lambda=3.2$ in minute.

According to the strategy-making process in chapter 3 , we will follow 3 steps to do the analysis.

Make an analysis on the demand charging power characteristics of 4500 electric cars and the local daily load-curve. According to the model we build in 2.1 and the Monte Carlo simulation, the power demand curve is shown as follows:

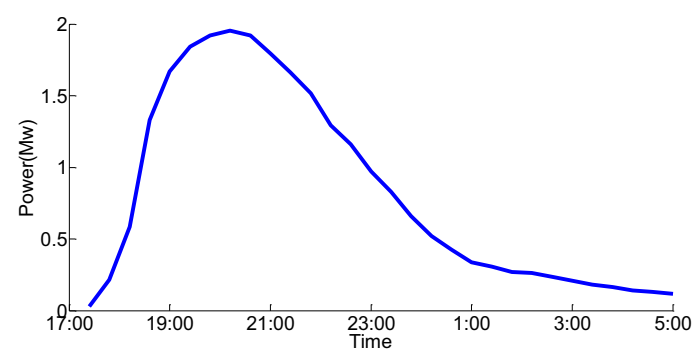

Figure 4. Power demand of Evs

According to the figure, we can obtain P1: 1.96MW.

The local daily load-curve and the load curve with electric vehicles are shown in Figure 5:

According to the figure, we can obtain that the local load peak value is $\mathrm{P}_{2}: 4.95 \mathrm{MW}$. The load with electric vehicles peak value is Pk: $6.70 \mathrm{MW}$.

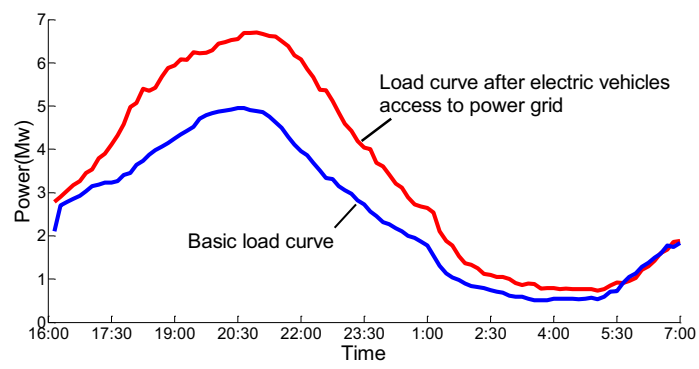

Figure 5. Local daily load-curve and load curve with electric vehicles

We can see that the $\mathrm{Pk}$ is less than $\mathrm{N}$-the capacity of distribution. According to the reference of Formula (7), $\mathrm{S}_{\max }=\mathrm{P}_{1} / \mathrm{K}=3920$.

The waiting time of users during peak hour will no more than $20 \mathrm{~min}$. Combined with formula (6)-(10), we can obtain that $S_{\min }: S_{\min }=2781.5$, and we will regard it as 2782 .

According to the analysis mentioned earlier, the deployment strategy for the electric vehicles in this area is the number which is suggested to range from 2782 to 3920 .

\section{CONCLUSIONS}

In this paper, the authors make the strategy which can serve the needs of users and ensure the safe operation of the distribution network at the same time. However, this paper can also give suggestions to the local distribution building plan. In the chapter 4 , the authors analyze the realistic use case, test and verify the analysis model in this paper, and adopt the specific strategy of the deployment. But in real life, there are lots of works need to do to obtain the accurate analysis model, because there are many factors which can influence the behavior of electric vehicles. The load curve of grid and the power of charging piles float rapidly. These factors influence the deployment for charging piles. There are lots of researches need to be developed in the future to solve the problem that how to plan the construction of charging facilities in order to make the system operates with high efficiency and do no harm to the operation of the grid.

\section{REFERENCES}

[1] Liting Tian, Shuanglong Shi. \& Zhuo Jia. 2010. Statistical modeling methods for electric vehicle charging power demands. Power System Technology, 34(11).

[2] Stanton W, H, Alexandra, A, Tsvetkova. 2009. Potential impacts of plug-in hybrid electric vehicles on regional power generation. The Electricity Journal, 22(10): 56-68. 
[3] Yongbiao Yang, Xiaohua Ding. \& Jinda Zhu. 2010. The Imagines about the Internet of Things used in electric vehicle charging facilities. Automation of Electric Power Systems, 34(11): 9598.

[4] Junhua Zhao, Fushuan Wen. \& Yusheng Xue. 2010. The stochastic economic dispatch which considering the uncertainties of electric vehicles and wind power output. Automation of Electric Power Systems, 34(20): 22-29.

[5] McCarthy D. \& Wolfs P. 2010. The HV system impacts of large scale electric vehicle deployments in a metropolitan area. 20th Australasian Universities Power Engineering Conference. Christchurch, Australia: University of Canterbury, pp: 1-6.

[6] Mei Huang. \& Shaofang Huang. 2008. The calculation method in engineering about the electric vehicle charging station harmonic. Power System Technology, 32(20): 20-23.

[7] Fengquan Zhou, Zhanwei Lian. \& Xiaolei, Wang. 2010 Analysis about the electric vehicle charging stations operating mode. Power System Protection and Control, 38(21): 63-66

[8] Ruisheng Li, Xiaolei Wang. \& Fengquan Zhou. 2010 Smart electric vehicle charging stations baesd on intelligent flow control. Power System Protection and Control, 38(21): 87-90

[9] Letian Teng, Weiguo He. \& Chenggang Du. 2009. EV energy supply model and its impact on the grid operations. East China Electric Power, 37(10): 1675-1677.

[10]Chunyang Wu, Canbing Li. \&Li Du. 2010. Planning methods for electric vehicle charging facilities. Automation of Electric Power Systems, 34(24): 36-39.

[11]Funian Hu, Yudong Tang. \& Yun Zhou. 2007. The analysis about the influence makes by implementation of TOU price policies on demand-side. Transactions of China Electro-technical Society, 22(4): 168-174.

[12]PUTRUS G, SUWANA Pingkarl A. \& JOHNSTON P. 2009. Impact ofelectric vehicles on power distribution networks. IEEE Vehicle Power and Propulsion Conference. 